\title{
The identification of sandfly species, from an area of Argentina with endemic leishmaniasis, by the PCR-based analysis of the gene coding for $18 \mathrm{~S}$ ribosomal RNA
}

\author{
P. A. BARROSO ${ }^{\star}$, J. D. MARCO ${ }^{\star}$, , H. KATO ${ }^{\ddagger}$, R. TARAMA ${ }^{\ddagger}$, P. RUEDA $^{\S}$, \\ S. P. CAJAL ${ }^{\S}$, M. A. BASOMBRÍO ${ }^{\dagger}, M$. KORENAGA ${ }^{\star}$, N. J. TARANTO ${ }^{\S}$ and \\ Y. HASHIGUCHI \\ *Department of Parasitology, Kochi Medical School, Kochi University, Kohasu, Okoh, Nankoku, \\ Kochi 783-8505, Japan \\ ${ }^{\dagger}$ Instituto de Patologia Experimental, Facultad de Ciencias de la Salud, Universidad Nacional de \\ Salta-Consejo Nacional de Investigaciones Cientificas y Técnicas, Buenos Aires 177, Salta 4400, \\ Argentina \\ ${ }^{\ddagger}$ Department of Veterinary Hygiene, Faculty of Agriculture, Yamaguchi University, Yamaguchi \\ 753-8515, fapan \\ ${ }^{\S}$ Instituto de Enfermedades Tropicales, Sede Regional Orán, Universidad Nacional de Salta, \\ Alvarado 751, San Ramón de la Nueva Orán, Salta 4530, Argentina
}

Received 10 February 2006, Revised 18 fuly 2006, Accepted 21 fuly 2006

\begin{abstract}
The area around Río Blanco, in the Orán department in the north of the Argentinian province of Salta, is endemic for American tegumentary leishmaniasis. In an attempt to facilitate the identification of the Lutzomyia species in this area, sequences of the gene coding for the $18 \mathrm{~S}$ ribosomal RNA (rRNA) of sandflies caught in a Shannon trap were explored, by a combination of PCR and analysis of restriction-fragment-length polymorphism (RFLP). The products from the PCR, which employed two primers developed specifically for this study (Lu.18S IS and Lu.18S $A R$ ), were cloned into a commercial vector (pGEM-T Easy) so that their nucleotide sequences could be investigated. In the RFLP analysis, the products of single and double digestion with the $A f a I$ and HapII restriction enzymes were separated by electrophoresis in $3 \%$ or $4 \%$ agarose. Taken together with the results of a morphological investigation of the flies, the resultant DNA fragment patterns were sufficient to identify most of the sandflies caught as $L u$. neivai. Although two other species, Lu. cortelezzii and $L u$. sallesi, were collected, they were relatively rare and only identified morphologically. A single digestion of the 18S-rRNA gene sequences with $A f a I$ or $H a p I I$ appeared sufficient and useful for the identification of $L u$. neivai from the north of Salta province, and for several other Lutzomyia species.
\end{abstract}

During the 1980 s and 1990 s, three epidemic outbreaks of American tegumentary leishmaniasis (ATL) occurred in the north of the Argentinian province of Salta, where Leishmania (Viannia) braziliensis is the predominant causative agent of the disease, followed by L. (Leishmania) amazonensis and $L$. (V.) guyanensis (Cupolillo et al.,

Reprint requests to: P. A. Barroso.; E-mail: paola@, med.kochi-u.ac.jp; fax: +8188 8802415 .
1994; Segura et al., 2000; Frank et al., 2003; Marco et al., 2005). Although five species of sandfly have been reported in the Orán and San Martín departments of Salta province - Lutzomyia neivai, Lu. migonei, Lu. cortelezzii, Lu. punctigeniculata and Lu. shannoni (Salomón et al., 2004) - little is known about their vectorial capacity. In most studies on the transmission of Leishmania spp., sandflies are identified by the morphological examination of internal 


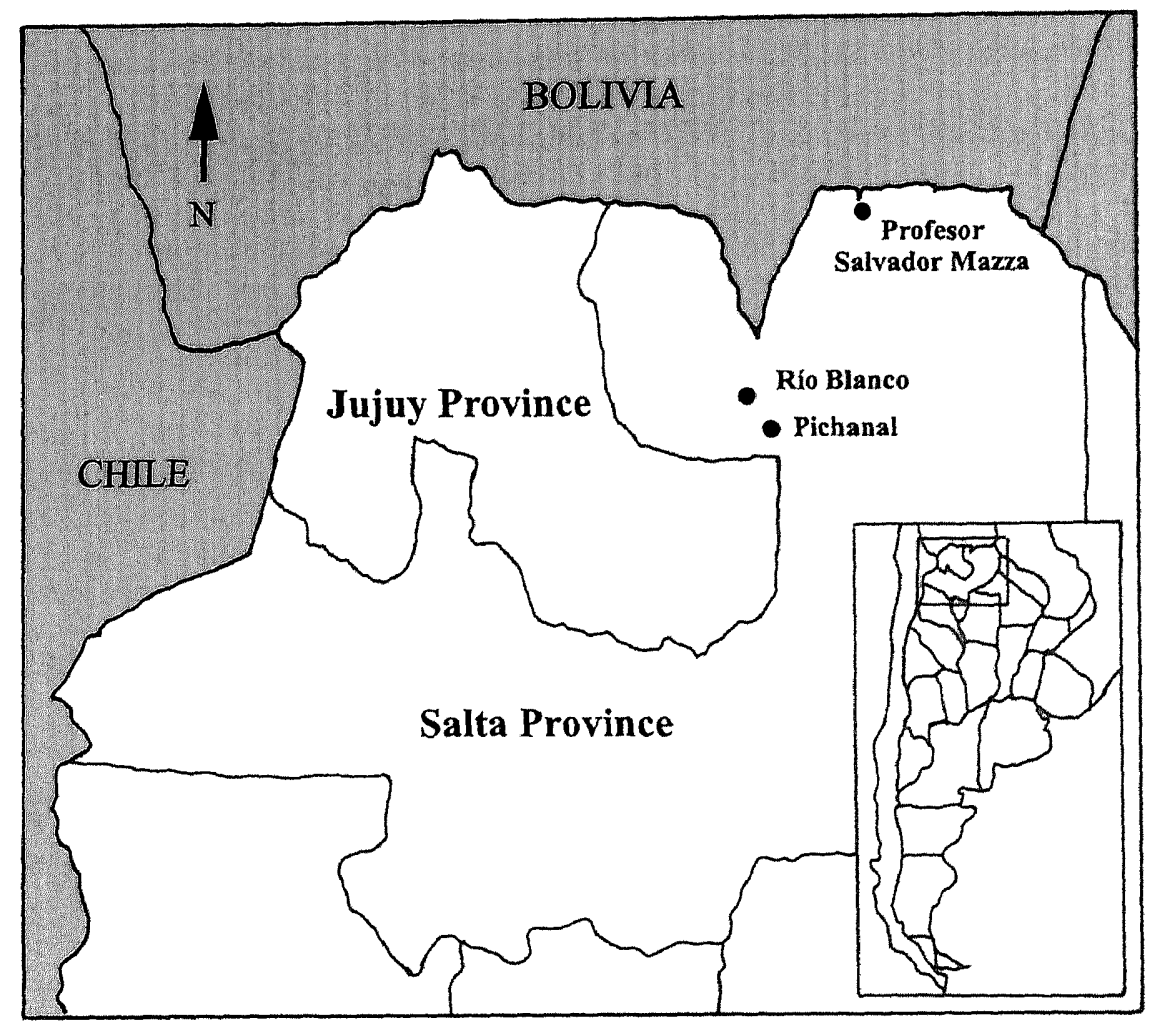

FIG. 1. Map of the north of Argentina, showing the locations of the sites, in Salta province, where the sandfly specimens were caught.

structures - often the spermathecae, cibaria and pharynges of the female flies and the terminal genitalia of the males (WHO, 1990). This technique, however, requires special taxonomic skills, is timeconsuming, cannot be applied simultaneously to two or more flies, and is often not useful in distinguishing between species that are morphologically very similar. Although iso-enzyme electrophoresis or the analysis of cuticular hydrocarbons by gas chromatography can be useful in identifying sandfly species, including those that are difficult to distinguish by morphology (WHO, 1990), they require refined storage conditions for the samples. Within the last decade, sandflies from the Old World have been successfully identified, to species level, by restriction-fragment-lengthpolymorphism (RFLP) analysis, using the products of the PCR-based amplification of the flies' 18S ribosomal RNA (rRNA) genes (Aransay et al., 1999). In the present study, this molecular technique, which appears to be reliable and does not need either refined storage conditions for the samples or taxonomic expertise, was applied, in a modified form, to the sandflies of northern Argentina.

\section{MATERIALS AND METHODS}

\section{Study Areas}

Sandflies were collected at two sites (A and $B$ ) in Río Blanco and at one site (C) in Pichanal, all in the Orán department, and at two sites (D and $E$ ) in Profesor Salvador Mazza, all in the northern part of Salta province, Argentina (Fig. 1). Cases of ATL have been reported at Río Blanco and Pichanal. Profesor Salvador Mazza has no recorded history of the disease, although it 
does possess phyto-geographical characteristics that appear favorable for sandflies (unpubl. obs.).

\section{Sandfly Collections}

The sandflies were collected, in an illuminated Shannon trap, during December 2002 and January 2003 (a period that coincides with the seasonal peak in the abundance of adult sandflies). Each collection was made between 20.30 to 22.30 hours, with the sandflies caught kept cool $\left(4^{\circ} \mathrm{C}\right)$ until the next morning, when most were dissected and identified by morphology. A subsample of the large collection made at site B in Río Blanco was fixed in $70 \%$ ethanol and stored until it could be investigated by PCR-RFLP (see below).

\section{Sandfly Species Identification}

MOLECULAR CLONING AND NUCLEOTIDE SEQUENCING OF SANDFLY 18S-RRNA GENE

Since the primers used by Aransay et al. (1999), in their studies of Old-World Phlebotomus, did not work on the NewWorld Lutzomyia collected in the present study, new primers, based on conserved sequences of the 18S-rRNA genes of Lutzomyia species, had to be developed. The new primers were named Lu.18S $1 \mathrm{~S}$ (5'-TGC CAG TAG TTA TAT GCT TG$\left.3^{\prime}\right)$ and Lu.18S AR (5'-CAC CTA CGG AAA CCT TGT TAC-3'). They were used in PCR similar to those described by Aransay et al. (1999), and each reaction used $25 \mu$ l of a commercial PCR mixture (Premix Taq ${ }^{\text {TM}}$; Takara Bio, Shiga, Japan) and DNA extracted (Aransay et al., 1999) from flies, from the site-B collection, that had either been identified, by morphology, as Lu. neivai or were 'unidentified'. The thermocycler used was set to give an initial denaturation at $95^{\circ} \mathrm{C}$ for $2 \mathrm{~min}$, followed by 40 cycles, each of denaturation at $95^{\circ} \mathrm{C}$ for $1 \mathrm{~min}$, annealing at $50^{\circ} \mathrm{C}$ for $1 \mathrm{~min}$, and polymerization at $72^{\circ} \mathrm{C}$ for $2 \mathrm{~min}$. The product of each PCR was cloned into a commercial vector ( $\mathrm{PGEM}-\mathrm{T}$ Easy Vector;
Promega, Madison, WI), and then sequenced (Kato et al., 2005). The levels of homology between the sequence of the PCR-amplified fragment from the flies morphologically identified as $L u$. neivai (GenBank accession number AB214970) and the 18S-rRNA genes from Lu. shannoni, Lu. geniculata, Lu. vattieri, Lu. toroensis, Lu. verrucarum and Lu. longipalpis were evaluated using the BLAST program (Altschul et al., 1990).

\section{RESTRICTION-FRAGMENT ANALYSIS}

For the RFLP analysis, $5 \mu 1$ of each PCR product were digested with the restriction enzymes AfaI (Takara Bio) and HapII (Takara Bio), which were used either separately or in combination. Each enzyme-digested sample was separated by electrophoresis in $3 \%$ or $4 \%$ agarose gel, to produce a DNA fragment pattern.

\section{MORPHOLOGICAL CHARACTERISTICS}

The morphological identification of the Lutzomyia caught was based mainly on the spermathecae and cibarial armatures, although, in some cases, it was also necessary to examine the male terminalia (Young and Duncan, 1994; Marcondes, 1996).

\section{RESULTS}

In the present study, in order to establish a molecular-genetics basis for the classification of Lutzomyia, analysis of the 18S-rRNA gene by PCR-RFLP was extended to samples from this New-World genus. The new primer pair, Lu.18S $1 S$ and Lu.18S $\mathrm{AR}$, amplified a single, identical DNA fragment, of about $2 \mathrm{kbp}$, from the total DNA of each of the site-B flies identified morphologically as Lu. neivai (Fig. 2) and that of each of the 19 unidentified sandflies from site $B$ that were also tested. The sequence of this PCR-amplified fragment showed $98 \%$ homology with the corresponding (18S-rRNA) genes from $\mathrm{Lu}$. 


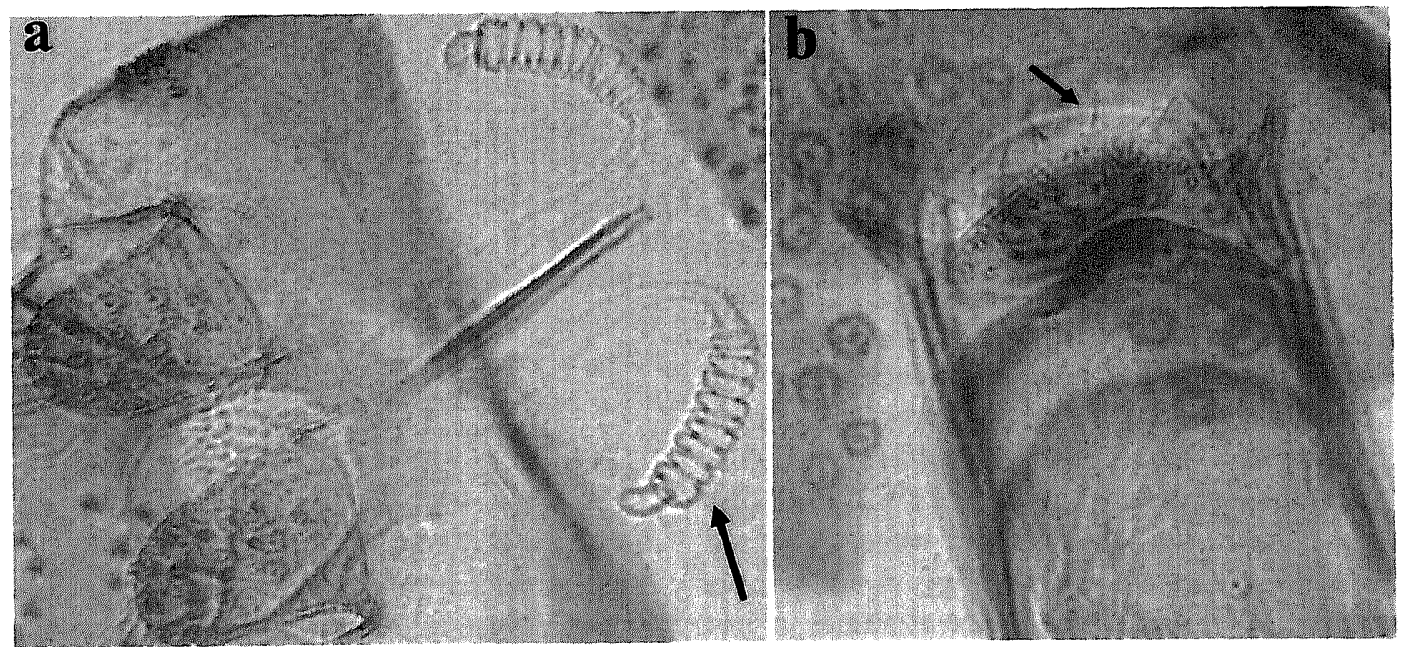

FIG. 2. Photomicrographs of a female Lutzomyia neivai, showing the spermathecae (a) and a horizontal tooth in the cibarium (b).

shannoni, Lu. geniculata, Lu. vattieri and $L u$. toroensis, and $97 \%$ homology with those from $L u$. verrucarum and $L u$. longipalpis. The $L u$. neivai 18S-rRNA gene sequence was split at seven points (nucleotide positions 50-51, 87-88, 529-530, 671-672, $863-864,1579-1580$ and $1864-1865)$ by $A f a I$, and at six points $(390-391,755-756$, $859-860, \quad 1225-1226, \quad 1249-1250$ and 1806-1807) by HapII. As shown in Figure 3(a), digestion of the PCR product with $A f a I$ alone therefore gave eight fragments (of $37,50,142,155,192,285,442$ and $716 \mathrm{bp}$ ), whereas digestion with HapII alone gave seven fragments (of 24, 104, 213, 365, 366, 390 and $557 \mathrm{bp}$ ). Double digestion of the $L u$. neivai 18S-rRNA gene sequence, with both $A f a \mathrm{I}$ and HapII, resulted in 14 fragments [of $4,24,37,50$, $58,84,104,139,142,155,227,303,330$ and 362 bp; Fig. 3(b)]. As the 19 'unidentified' sandflies from Río Blanco gave fragment patterns similar to that produced using the morphologically identified $L u$. neivai, they were all identified as this species [Fig. 3(b)].

Usefully, in terms of taxonomy, the same PCR-RFLP analysis, using single digestion with either $A f a \mathrm{I}$ or HapII and DNA from Lu. shannoni, Lu. geniculata, Lu. vattieri,
Lu. toroensis, Lu. verrucarum, Lu. longipalpis or any of several other Lutzomyia species, always gave a species-specific pattern that was distinct from that of $\mathrm{Lu}$. neivai and that of any other species tested (data not shown).

Almost all the sandflies collected in the present study were identified as $L u$. neivai (see Table). The samples of the other species collected, which were identified by morphology as $L u$. cortelezzii and Lu. sallesi, were judged too small to make their investigation by PCR-RFLP worthwhile (Table).

\section{DISCUSSION}

In the present study, attempts were made to modify and standardize the ' $18 \mathrm{~S}-\mathrm{rRNA}$ gene' technique developed for Phlebotomus in the Old World (Aransay et al., 1999), for use in the identification of Argentinian Lutzomyia (initially those caught at Río Blanco, in the department of Orán, where ATL is endemic). Since PCR based on the $\mathrm{F} 1$ and $\mathrm{R} 1$ primers developed by Aransay et al. (1999) did not give any useful product when run with Lutzomyia DNA (data not shown), a new pair of primers, Lu.18S $1 \mathrm{~S}$ 
a 12 3
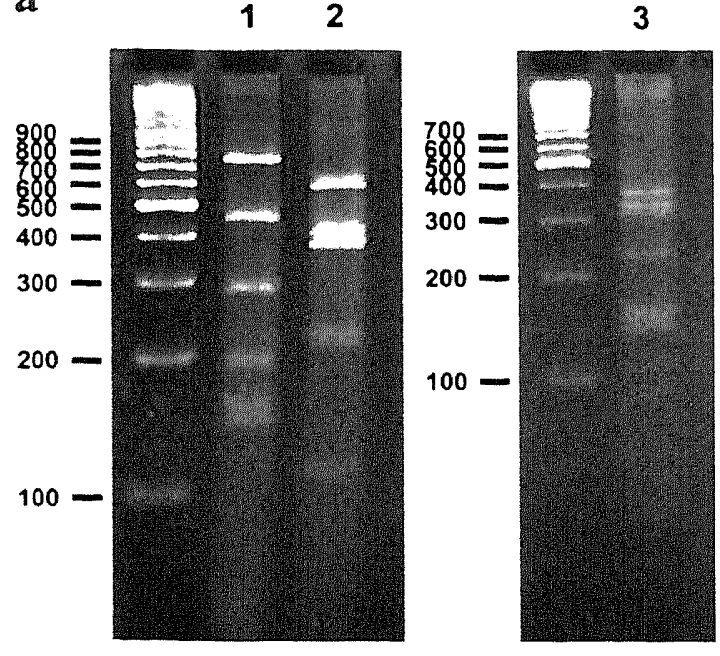

b
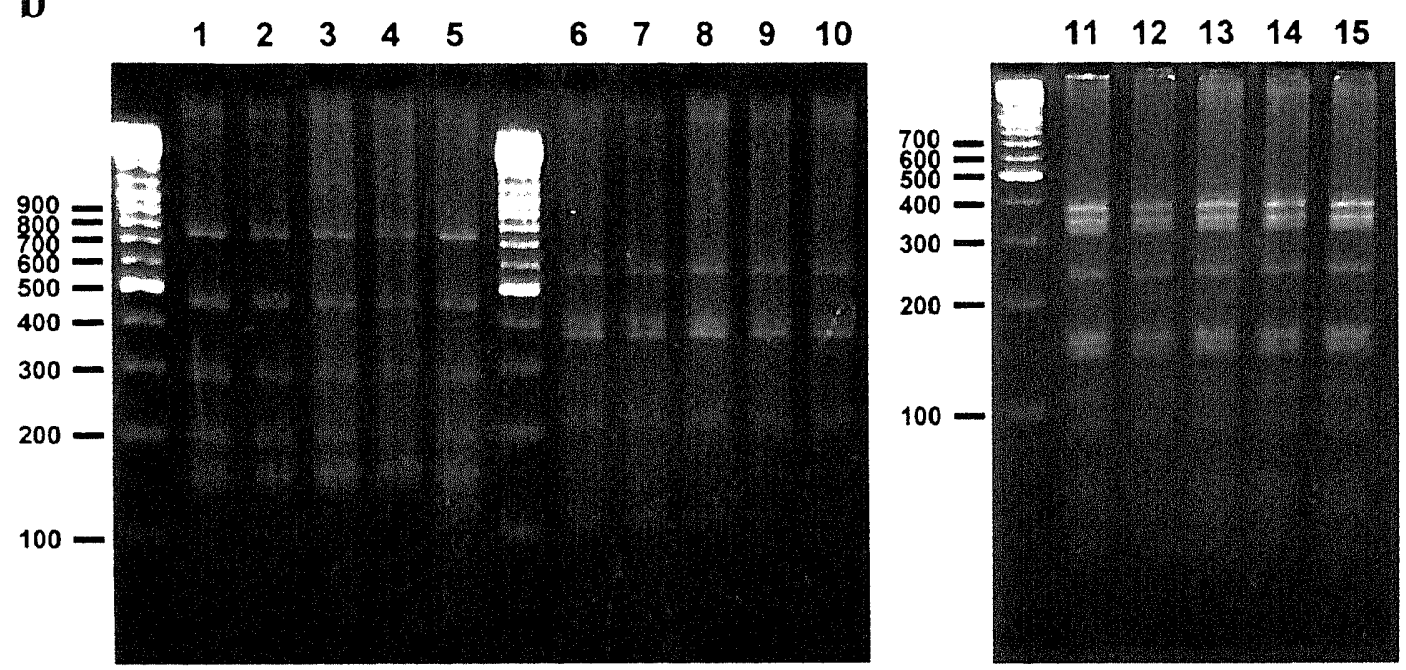

FIG. 3. Classification of Argentinian Lutzomyia by the PCR-RFLP analysis of their 18S-rRNA gene fragments. Panel (a) shows the fragments, from a fly morphologically identified as $L u$. neivai, produced by digestion with $A f a I$ (lane 1), HapII (lane 2) or both $A f a I$ and HapII (lane 3). Panel (b) illustrates how five of the 19 unidentified flies showed identical fragment patterns after the PCR-amplified fragment of their 18S-rRNA genes was digested with AfaI alone (lanes 1-5), HapII alone (lanes 6-10), or the combination of both of these enzymes (lanes 11-15); the other 14 unidentified flies showed the same patterns.

and Lu.18S AR, had to be produced. By using this pair of primers, a fragment of the Lu. neivai 18S-rRNA gene could be amplified and compared with the corresponding sequences of Lu. shannoni, Lu. geniculata, Lu. vattieri, Lu. toroensis, Lu. verrucarum and Lu. longipalpis, which showed 97\%-98\% homology. Usefully, at least for Lu. neivai from Salta province and several other species of Lutzomyia, the interspecific differences in the fragments amplified in PCR based on the Lu.18S $1 \mathrm{~S}$ and Lu.18S AR primers were sufficient to give speciesspecific patterns when those products were digested with $A f a \mathrm{I}$ or HapII and then subjected to electrophoresis. 
TABLE 1. The results of the morphological or molecular identification, to species level, of the sandfies collected in Salta province, Argentina, in December 2002-fanuary 2003

\begin{tabular}{l}
\hline \\
\cline { 2 - 6 } \\
\cline { 2 - 5 }
\end{tabular}

Although, in the PCR-RFLP study of Old-World sandflies by Aransay et al. (1999), double digestion, with RsaI (which cleaves the same sites as $A f a \mathrm{I}$ ) and $H p a I I$ (which cleaves the same sites as HapII), sometimes gave more useful results than digestion with a single restriction enzyme, this was not the case with the Argentinian Lutzomyia (present study). Aransay et al. (1999) found that double digestion gave species-specific patterns for $P$. papatasi, $P$. alexandri, $P$. sergenti, $P$. simici, Sergentomyia minuta, $S$. fallax cypriotica and $S$. dentata, although three other Phlebotomus species, all belonging to the subgenus Larroussius, required a second double digestion, with $A c c I$ and BanI, to distinguish them. In the present study, double digestion, with $A f a I$ and HapII, gave many, relatively small fragments and a fragment pattern that was, for the identification of the species investigated, no more useful and slightly harder to 'read' than the pattern produced when either of these enzymes was used alone.

In conclusion, the present results indicate that PCR-RFLP analysis of the 18S-rRNA gene could be a suitable and useful method for the classification of the Lutzomyia species found in the north of Salta province. The usefulness of this technique in the identification of other Lutzomyia species, from Salta province and elsewhere, will be explored in future studies.

ACKNOWLEDGEMENTS. The authors thank D. B. Ribble, for his constructive comments on the manuscript. This study was supported financially by the Ministry of Education, Science, Culture and Sports of Japan (via grants 14256002 and 18256004).

\section{REFERENCES}

Altschul, S. F. , Gish, W., Miller, W., Myers, E. W. \& Lipman, D. J. (1990). Basic local alignment search tool. Fournal of Molecular Biology, 215, 403-410.

Aransay, A. M., Scoulica, E., Chaniotis, B. \& Tselentis, $Y$. (1999). Typing of sandflies from Greece and Cyprus by DNA polymorphism of $18 \mathrm{~S}$ rRNA gene. Insect Molecular Biology, 8, 179-184.

Cupolillo, E., Grimaldi Jr, G. \& Momen, H. (1994). A general classification of New World Leishmania using numerical zymotaxonomy. American Fournal of Tropical Medicine and Hygiene, 50, 296-311.

Frank, F. M., Fernández, M. M., Taranto, N.J., Cajal, S. P., Margni, R. A., Castro, E., Thomaz-Soccol, V. \& Malchiodi, E. L. (2003). Characterization of human infection by Leishmania spp. in the northwest of Argentina: immune response, double infection with Trypanosoma cruzi and species of Leishmania involved. Parasitology, 126, 31-39.

Kato, H., Uezato, H., Katakura, K., Calvopiña, M., Marco, J. D., Barroso, P. A., Gomez, E. A. L., Mimori, T., Korenaga, M., Iwata, H., Nonaka, S. \& Hashiguchi, Y. (2005). Detection and identification 
of Leishmania species within naturally infected sand flies in the Andean areas of Ecuador by a polymerase chain reaction. American foumal of Tropical Medicine and Hygiene, 72, 87-93.

Marco, J. D., Barroso, P. A., Calvopiña, M., Kumazawa, H., Furuya, M., Korenaga, M., Cajal, S. P., Mora, M. C., Rea, M. M. J., Borda, C. E., Basombrio, M. A., Taranto, N. J. \& Hashiguchi, Y. (2005). Species assignation of Leishmania from humans and canine American tegumentary leishmaniasis cases by multilocus enzyme electrophoresis in north Argentina. American foumal of Tropical Medicine and Hygiene, 72, 606-611.

Marcondes, C. B. (1996). A redescription of Lutzomyia (Nyssomyia) intermedia (Lutz \& Neiva, 1912), and resurrection of $L$. neivai (Pinto, 1926) (Diptera, Psychodidae, Phlebotominae). Memórias do Instituto Oswaldo Cruz, 91, 457-462.

Salomón, O. D., Wilson, M. L., Munstermann, L. E. \& Travi, B. L. (2004). Spatial and temporal patterns of phlebotomine sand flies (Diptera: Psychodidae) in a cutaneous leishmaniasis focus in northern Argentina. Fournal of Medical Entomology, 41, 3339.

Segura, E. L., Juan, N., Piquin, A. L. M., Cuba-Cuba, C. A., Abramo-Orrego, L., McMahon-Pratt, D., Montamat, E. E., Momen, H. \& Grimaldi Jr, G. (2000). Molecular and biologic characterization of Leishmania parasites implicated in an epidemic outbreak in northwestern Argentina. Parasitology Research, 86, 504-508.

World Health Organization. (1990). Control of the Leishmaniases. Technical Report Series No. 793. Geneva: WHO.

Young, D. G. \& Duncan, M. A. (1994). Guide to the identification and geographic distribution of Lutzomyia sand flies in Mexico, the West Indies, Central and South America (Diptera: Psychodidae). Memoirs of the American Entomological Institute, 54, 1881 . 